\section{Dr. Malaviya, et al reply}

\section{To the Editor:}

Kharbanda, et al report development of tuberculosis (TB) infection in 2 of their patients, 3 weeks and 3 months after the last dose of infliximab, despite following the LTBI screening strategy suggested in our publication ${ }^{1}$. One of their patients was in a pediatric age group. We had also reported post infliximab TB infection in one of our patients despite following the latent TB infection (LTBI) screening strategy that we recommended ${ }^{1}$. We had, therefore, cautioned vigilance against TB flare especially in high TB-burden countries like India. Observations of Kharbanda, et al add weight to our argument.

Also, it is to be noted that the LTBI screening strategy that we recommended is only for adults, as we did not study children. The strategy may or may not be applicable in children and needs further work. Regarding the adult patient reported by Kharbanda, et al, the dose of tuberculin used for the tuberculin skin test (TST) was not mentioned. It is important to use 10 tuberculin units for TST to "break through" the relative anergy known in $\mathrm{RA}^{1}$. It is the single most important point of our paper and needs to be followed stringently.

Of course all the other standard methodological details for TST, including appropriate standardized preparation of purified protein derivative (PPD), must be followed for satisfactory performance of the test. Since our original report, we have used a similar LTBI screening strategy in another 33 patients given infliximab. We did not encounter the problem of TB flare in any of them ${ }^{2}$. Whether using ELISpot test would improve the sensitivity for detecting LTBI remains to be established.

We feel that clinical vigilance and recurrent testing are better strategies than continuing longterm therapy in patients once the underlying presumed infection has been treated, thus decreasing the likelihood of toxicity from longterm continual use and, potentially, allowing emergence of resistant strains.

ANAND N. MALAVIYA, MD, FRCP, MACR, A\&R Clinic for Arthritis and Rheumatism, ISIC Superspeciality Hospital - Rheumatology, New Delhi; SANJIV KAPOOR; SHRIRAM GARG; ROOPA RAWAT, ISIC

Superspeciality Hospital - Rheumatology, New Delhi; SUBRAMANIAN SHANKAR, Internal Medicine, Division of Rheumatology and Clinical Immunology, Armed Forces Medical College, Pune, Maharashtra; SAVITA NAGPAL, Dr. Savita Nagpal Laboratory, New Delhi; DINESH KHANNA, David Geffen School of Medicine, University of California, Los Angeles, Los Angeles, California, USA. Address correspondence to Dr. A.N. Malaviya; E-mail: anand_malaviya@yahoo.com

\section{REFERENCES}

1. Malaviya AN, Kapoor S, Garg S, Rawat R, Shankar S, Nagpal S, et al. Preventing tuberculosis flare in patients with inflammatory rheumatic diseases receiving tumor necrosis factor-alpha inhibitors in India - An audit report. J Rheumatol 2009;36:1414-20.

2. Malaviya AN, Kapoor S, Garg S, Rawat R. Recommended screening strategy for preventing tuberculosis flare in patients with inflammatory rheumatic diseases receiving tumor necrosis factor-alpha inhibitors in India - followup report. J Rheumatol 2010;37:209.

J Rheumatol 2010;37:7; doi:10.3899/jrheum.100175 